JOURNAL OF THE

AMERICAN MATHEMATICAL SOCIETY

Volume 16, Number 2, Pages 285-302

S 0894-0347(02)00414-9

Article electronically published on November 27, 2002

\title{
REGULARITY ON ABELIAN VARIETIES I
}

\author{
GIUSEPPE PARESCHI AND MIHNEA POPA
}

\section{INTRODUCTION}

This is the first in a series of papers meant to introduce a notion of regularity on abelian varieties and more general irregular varieties. This notion, called Mukai regularity, is based on Mukai's concept of Fourier transform, and in a very particular form (called Theta regularity) it parallels and strengthens the usual CastelnuovoMumford regularity with respect to polarizations on abelian varieties. Mukai regularity turns out to have a large number of applications, ranging from basic properties of linear series on abelian varieties and defining equations for the ideals of their subvarieties, to higher dimensional type statements and to a study of special classes of vector bundles. As a quite surprising example, one obtains statements of a very classical flavor about the ideals of special subvarieties in Jacobians. Some of these applications are explained in the present paper, while others, together with the necessary theoretical refinements, are the subject of the sequels [PP1, [PP2].

Let $X$ be an abelian variety of dimension $g$ over an algebraically closed field. Denote by $\hat{X}$ its dual, and let $\mathcal{P}$ be a Poincaré line bundle on $X \times \hat{X}$, properly normalized. The Fourier-Mukai functor is the derived functor associated to the functor which takes a coherent sheaf $\mathcal{F}$ on $X$ to $\hat{\mathcal{S}}(\mathcal{F}):=p_{\hat{X} *}\left(p_{X}^{*} \mathcal{F} \otimes \mathcal{P}\right)$ on $\hat{X}$. We define Mukai regularity to be a condition on the cohomologies of the derived complex, weaker than the usual Index Theorem or Weak Index Theorem of [M1]. Concretely, a sheaf $\mathcal{F}$ on $X$ will be called Mukai regular, or simply $M$-regular, if

$$
\operatorname{codim}\left(\operatorname{Supp} R^{i} \hat{\mathcal{S}}(\mathcal{F})\right)>i, \forall i>0 \text {. }
$$

Recall that a sheaf $\mathcal{F}$ is said to satisfy the Index Theorem (I.T.) with index 0 if

$$
h^{i}(\mathcal{F} \otimes \alpha)=0, \forall \alpha \in \operatorname{Pic}^{0}(X), \forall i>0 ;
$$

thus sheaves satisfying I.T. with index 0 are trivially $M$-regular. The main theme in what follows is that $M$-regularity has significant geometric consequences and can be verified in practice in a variety of situations. As in many other settings, geometric information is obtained via the global generation of suitably chosen sheaves, as we will see repeatedly below. This principle takes various forms, and the following is the main and most commonly used in this paper.

Theorem ( $M$-regularity criterion). Let $\mathcal{F}$ be a coherent sheaf and $L$ an invertible sheaf supported on a subvariety $Y$ of the abelian variety $X$ (possibly $X$ itself). If both $\mathcal{F}$ and $L$ are $M$-regular as sheaves on $X$, then $\mathcal{F} \otimes L$ is globally generated.

Received by the editors October 22, 2001 and, in revised form, April 4, 2002.

2000 Mathematics Subject Classification. Primary 14K05; Secondary 14K12, 14H40, 14 E05.

The second author was partially supported by a Clay Mathematics Institute Liftoff Fellowship during the preparation of this paper. 
By fixing a polarization $\Theta$ on $X$, one obtains a corollary which itself provides a wide range of applications when combined with various vanishing theorems. It is a generalization of a result on vector bundles proved in $\mathrm{Pa}$, Theorem 2.1]. Loosely speaking, it is precisely this extension to arbitrary coherent sheaves which allows one to attack a number of problems related to ideal sheaves or sheaves supported on subvarieties of $X$.

Corollary (Theta-regularity criterion). Let $\mathcal{F}$ be a coherent sheaf on the polarized abelian variety $(X, \Theta)$. If $\mathcal{F}$ is $\Theta$-regular, i.e., $\mathcal{F}(-\Theta)$ is $M$-regular, then $\mathcal{F}$ is globally generated. In particular, if $Y$ is a subvariety of $X$ such that $\mathcal{I}_{Y}(m \Theta)$ is $\Theta$-regular, then $\mathcal{I}_{Y}$ is cut out by divisors linearly equivalent to $m \Theta$.

Based on a more refined notion of continuous global generation, we will see in fact that in the above the stronger fact that $\mathcal{I}_{Y}$ is cut out by divisors algebraically equivalent to $(m-1) \Theta$ also holds. The corollary above, and other Theta-regularity results, are collected in $\S 6$ in Theorem 6.3, which can be seen as an abelian analogue of the well-known Castelnuovo-Mumford Lemma.

The $M$-regularity criterion is in turn a consequence of the following statement, which is the main technical result of the paper. The theorem is an extended generalization of a result on vector bundles appearing in various forms in work of Mumford, Kempf and Lazarsfeld (cf. [Ke2] or [Ke3]) and is explained in detail in Pa. The proof makes essential use of the full extent of Mukai's Fourier transform methods in a derived category setting.

Theorem. Let $\mathcal{F}$ and $H$ be sheaves on $X$ such that $\mathcal{F}$ is $M$-regular and $H$ is locally free satisfying I.T. with index 0. Then, for any nonempty Zariski open set $U \subset \hat{X}$, the map

$$
\mathcal{M}_{U}: \bigoplus_{\xi \in U} H^{0}\left(X, \mathcal{F} \otimes P_{\xi}\right) \otimes H^{0}\left(X, H \otimes P_{\xi}^{\vee}\right) \stackrel{\oplus m_{\xi}}{\longrightarrow} H^{0}(X, \mathcal{F} \otimes H)
$$

is surjective, where $m_{\xi}$ denote the multiplication maps on global sections.

Turning to applications, it is interesting to note in the first place that the apparently involved machinery of $M$-regularity has in fact consequences of a very elementary nature. The main application of this kind in the present paper is to the cohomological properties and the equations defining the images $W_{d}$ of symmetric products of a curve $C$ in its polarized Jacobian $(J(C), \Theta)$, via Abel-Jacobi mappings. The intuitive reason for the need of such methods is that naturally defined sheaves, like the restriction $\mathcal{O}_{W_{d}}(\Theta)$, are Mukai-regular, while not having better cohomological properties (cf. $\S 4$ ).

Theorem. For any $1 \leq d \leq g-1$, the twisted ideal sheaf is 3 - $\Theta$-regular, more precisely $\mathcal{I}_{W_{d}}(2 \Theta)$ satisfies $I . T$. with index 0 .

Corollary. For any $1 \leq d \leq g-1$, the ideal $\mathcal{I}_{W_{d}}$ is cut out by divisors algebraically equivalent to $2 \Theta$. It is also cut out by divisors linearly equivalent to $3 \Theta$.

As far as we are aware, the statement of the corollary seems to be new even in the case $i=1$, i.e., for an Abel-Jacobi embedding of $C$ in its Jacobian. Another immediate consequence is an affirmative answer to Conjecture 10.3 in [OP], on the sections of $2 \Theta$ on symmetric products.

Corollary (Oxbury-Pauly conjecture). In the notation above, the following are true: 
(1) $h^{0}\left(C^{d}, u_{d}^{*} \mathcal{O}(2 \Theta)\right)=\Sigma_{i=0}^{d}\left(\begin{array}{c}g \\ i\end{array}\right)$.

(2) $u_{d}^{*}: H^{0}(J(C), \mathcal{O}(2 \Theta)) \rightarrow H^{0}\left(C^{d}, u_{d}^{*} \mathcal{O}(2 \Theta)\right)$ is surjective.

In a rather different direction, in combination with Kawamata-Viehweg, Kollár or Nadel vanishing type theorems (cf. La1), our regularity criteria produce applications with a higher dimensional flavor. In this paper we take only the first steps in this direction, by looking at subvarieties of abelian varieties and their finite covers (but cf. also Remark 5.3), while a more general study will be developed in PP2. We collect in the following theorem some of the consequences obtained here (cf. $\S 5$ for these and further results).

Theorem. (i) (Finite Albanese maps) Let $Y$ be a smooth irregular variety whose Albanese map is finite onto its image and let $L$ be a big and nef line bundle on $Y$. Then $\omega_{Y}^{\otimes 2} \otimes L^{\otimes 2}$ is globally generated. In particular, if $Y$ is in addition a minimal variety of general type, then $\omega_{Y}^{\otimes 4}$ is globally generated. Moreover, if $\Theta$ is a polarization on $\operatorname{Alb}(Y)$, then $\omega_{Y} \otimes L \otimes \operatorname{alb}_{Y}^{*} \mathcal{O}(\Theta)$ is globally generated.

(ii) (Generalized Lefschetz Theorem) Under the same hypotheses, $\omega_{Y}^{\otimes 3} \otimes L^{\otimes 3}$ is very ample. In particular, if $Y$ is also a minimal variety of general type, then $\omega_{Y}^{\otimes 6}$ is very ample.

(iii) (Relative base point freeness) Let $f: Y \rightarrow X$ be a proper, surjective morphism from a smooth variety $Y$ to an abelian variety $X$, and denote by $\omega_{Y}$ the canonical line bundle on $Y$. Then $f_{*} \omega_{Y} \otimes \mathcal{O}_{X}(2 \Theta)$ is globally generated, for any ample divisor $\Theta$ on $X$.

(iv) (Mumford Lemma for multiplier ideals) Let $D$ be an effective $\mathbb{Q}$-divisor on a polarized abelian variety $(X, \Theta)$ and let $\mathcal{J}(D)$ be its associated multiplier ideal sheaf. If $L$ is a divisor on $X$ such that $L-D$ is ample, then the sheaf $\mathcal{O}_{X}(\Theta+L) \otimes$ $\mathcal{J}(D)$ is globally generated.

The reader not familiar with the topic will find an explanation of the significance of (iv) above in $\S 6$. Parts (i) and (ii) are based on the fact that $\omega_{Y} \otimes L$ has an $M$-regular (in fact I.T.0) push-forward to the Albanese variety, which will also imply that $H^{0}\left(\omega_{Y} \otimes L\right) \neq 0$. This answers positively a nonvanishing conjecture of Kawamata [Ka2] in the case of varieties of this kind (cf. Corollary 5.4).

As one of the main applications of this theory, the concept of $M$-regularity allows for a unified point of view in the study of linear series on abelian varieties and their subvarieties. Here we discuss first cases of this assertion - for example, it is interesting to note that the classical Lefschetz and Ohbuchi very ampleness theorems and the fact that line bundles of degree $2 g+1$ or more on curves are very ample are simply instances of the same phenomenon of producing $M$-regularity from numerical hypotheses (cf. $\S 4$ ). This can now be extended to a variety of numerical statements on arbitrary varieties which can be embedded in abelian varieties (for instance on $W_{d}$ 's, to refer to the most direct generalization of the case of curves).

Restricting the present discussion to linear series on abelian varieties, based on the methods of this paper and those of [Pa], in the sequel [PP1] we will show among other things the following, improving on the well-known Lefschetz-OhbuchiMumford-Kempf-Koizumi type results:

Theorem. (i) If $L$ is an ample line bundle on $X$ with $M$-regularity index $m(L)$, then $L^{\otimes(k+2-m(L))}$ is k-jet ample.

(ii) If $L$ is an ample line bundle with no base divisor on $X$, then the ideal of $X$ is cut out by quadratic equations in the embedding given by $L^{\otimes k}, k \geq 3$. 
(iii) Under the same hypothesis, the ideal of $X$ is cut out by quadratic and cubic equations in the embedding given by $L^{\otimes 2}$ (note that this is an embedding by a theorem of Ohbuchi).

The $M$-regularity index is a new invariant introduced in [PP1]; we will not give the definition here. Note only that if $L$ has no base divisor, then $m(L) \geq 1$. Statements (ii) and (iii) can be extended to arbitrary higher syzygies and also related to the invariant $m(L)$, improving on a conjecture of Lazarsfeld proved in $\mathrm{Pa}$. In fact it will be seen in [PP1 that essentially all the results on embeddings, higher order properties and defining equations for multiples of ample line bundles existing in the literature, plus new results like the theorem above, can be subsumed in a more general framework depending on these invariants defined via $M$-regularity.

Finally, it is important to emphasize that some of the underlying ideas in this work have separately existed in one form or another for quite some time. All our constructions rely heavily on Mukai's remarkable theory of the Fourier functor. On the other hand, it was Kempf who first realized that results in the style of Theorem 2.5 provide a theta-group-free approach to statements on linear series, based on vanishing theorems for vector bundles. The new ingredients brought in by the present approach are the inclusion of arbitrary coherent sheaves in the general study, the systematic use of cohomological criteria for the global generation of such sheaves, and the relaxation of strong vanishing conditions to the weaker $M$-regularity, all of which largely extend the range of applications. It is here that this paper claims its main originality.

The paper is structured as follows. In Section 2 we introduce the basic definitions and prove the general $M$-regularity and multiplication criteria. Section 3 is devoted to a series of examples and first applications. Sections 4 and 5 contain the main applications of the paper. In Section 4 we study the cohomological properties and the equations defining the $W_{d}$ 's in Jacobians, including the proof of the OxburyPauly conjecture. In Section 5 we give various effective results for linear series on subvarieties of abelian varieties and more general irregular varieties. Section 6 we restrict ourselves to a discussion of Theta regularity, drawing on a comparison with the usual Castelnuovo-Mumford regularity.

Background and notation. In what follows, unless otherwise specified, $X$ will be an abelian variety of dimension $g$ over an algebraically closed field. We will always denote arbitrary coherent sheaves on $X$ with script letters (e.g., $\mathcal{F}$ ) and locally free sheaves with straight letters (e.g., $F$ ). Given a line bundle $L$, we write $B(L)$ for the base locus of the linear series $|L|$.

We denote by $\hat{X}$ the dual abelian variety, which will often be identified with $\operatorname{Pic}^{0}(X)$. Given a point $\xi \in \hat{X}$, by $P_{\xi} \in \operatorname{Pic}^{0}(X)$ we understand the corresponding line bundle via this identification. By $\mathcal{P}$ we denote a Poincaré line bundle on $X \times \hat{X}$, normalized such that $\mathcal{P}_{\mid X \times\{0\}}$ and $\mathcal{P}_{\mid\{0\} \times \widehat{X}}$ are trivial.

We briefly recall the Fourier-Mukai setting, referring to [M1] for details. To any coherent sheaf $\mathcal{F}$ on $X$ we can associate the sheaf $p_{2 *}\left(p_{1}{ }^{*} \mathcal{F} \otimes \mathcal{P}\right)$ on $\widehat{X}$, where $p_{1}$ and $p_{2}$ are the natural projections on $X$ and $\hat{X}$. This correspondence gives a functor $\hat{\mathcal{S}}: \operatorname{Coh}(X) \rightarrow \operatorname{Coh}(\widehat{X})$. If we denote by $\mathbf{D}(X)$ and $\mathbf{D}(\hat{X})$ the derived categories of

\footnotetext{
${ }^{1}$ We have recently found out about the paper [CH] which has, among other things, an overlap with some of the results of this section.
} 
$\operatorname{Coh}(X)$ and $\operatorname{Coh}(\hat{X})$, then the derived functor $\mathbf{R} \hat{\mathcal{S}}: \mathbf{D}(X) \rightarrow \mathbf{D}(\hat{X})$ is defined and called the Fourier functor, and one can consider $\mathbf{R} \mathcal{S}: \mathbf{D}(\hat{X}) \rightarrow \mathbf{D}(X)$ in a similar way. Mukai's main result [M1, Theorem 2.2] is that $\mathbf{R} \hat{\mathcal{S}}$ establishes an equivalence of categories between $\mathbf{D}(X)$ and $\mathbf{D}(\hat{X})$.

Let $R^{j} \hat{\mathcal{S}}(\mathcal{F})$ be the cohomologies of the derived complex $\mathbf{R} \hat{\mathcal{S}}(\mathcal{F})$. A coherent sheaf $\mathcal{F}$ on $X$ satisfies W.I.T. (the weak index theorem) with index $i$ if $R^{j} \hat{\mathcal{S}}(\mathcal{F})=0$ for all $j \neq i$. It satisfies the stronger I.T. (the index theorem) with index $i$ if $H^{i}(\mathcal{F} \otimes \alpha)=0$ for all $\alpha \in \operatorname{Pic}^{0}(X)$ and all $i \neq j$. By the base change theorem, in this situation $R^{j} \hat{\mathcal{S}}(\mathcal{F})$ is locally free. If $\mathcal{F}$ satisfies W.I.T. with index $i, R^{i} \hat{\mathcal{S}}(\mathcal{F})$ is denoted by $\hat{\mathcal{F}}$ and is called the Fourier transform of $\mathcal{F}$. Note that then $\mathbf{R} \hat{\mathcal{S}}(\mathcal{F}) \cong$ $\hat{\mathcal{F}}[-i]$.

\section{Mukai Regularity, global generation AND CONTINUOUS GLOBAL GENERATION}

Let $X$ be an abelian variety of dimension $g$. Given a coherent sheaf $\mathcal{F}$ on $X$, we denote

$$
S^{i}(\mathcal{F}):=\operatorname{Supp}\left(R^{i} \hat{\mathcal{S}}(\mathcal{F})\right)
$$

Definition 2.1. A coherent sheaf $\mathcal{F}$ on $X$ is called Mukai-regular (or simply $M$ regular) if $\operatorname{codim}\left(S^{i}(\mathcal{F})\right)>i$ for any $i=1, \ldots, g$ (where, for $i=g$, this means that $S^{g}(\mathcal{F})$ is empty).

Example 2.2. Sheaves satisfying I.T. with index 0 are the simplest examples of $M$-regular sheaves. A first important class is that of ample line bundles on $X$.

Remark 2.3. From the definition of the Fourier transform we see that there is always an inclusion $S^{i}(\mathcal{F}) \subset V^{i}(\mathcal{F})$, where $V^{i}(\mathcal{F})$ is the cohomological support locus (cf. GL]):

$$
V^{i}(\mathcal{F}):=\left\{\xi \mid h^{i}\left(\mathcal{F} \otimes P_{\xi}\right) \neq 0\right\} \subset \operatorname{Pic}^{0}(X) .
$$

Consequently, $M$-regularity is achieved if in particular

$$
\operatorname{codim}\left(V^{i}(\mathcal{F})\right)>i \quad \text { for any } i=1, \ldots, g .
$$

It will be this property that we will usually check in applications.

The key point in what follows is that $M$-regularity is a cohomological condition which has significant geometric consequences via the global generation of suitable sheaves. The main result is the following:

Theorem 2.4. Let $\mathcal{F}$ be a coherent sheaf and $L$ an invertible sheaf supported on a subvariety $Y$ of the abelian variety $X$ (possibly $X$ itself). If both $\mathcal{F}$ and $L$ are $M$-regular as sheaves on $X$, then $\mathcal{F} \otimes L$ is globally generated.

The proof of Theorem 2.4 essentially occupies the rest of this section; more precisely, it is a formal consequence of Proposition 2.12 and Proposition 2.13 below. The key technical theorem, for which we will also find independent use in later sections, generalizes in several ways a result of Mumford-Kempf-Lazarsfeld explained in $[\mathrm{Pa}, \S 2]$. 
Theorem 2.5. Let $\mathcal{F}$ and $H$ be sheaves on $X$ such that $\mathcal{F}$ is $M$-regular and $H$ is locally free satisfying I.T. with index 0. Then, for any nonempty Zariski open set $U \subset \hat{X}$, the map

$$
\mathcal{M}_{U}: \bigoplus_{\xi \in U} H^{0}\left(X, \mathcal{F} \otimes P_{\xi}\right) \otimes H^{0}\left(X, H \otimes P_{\xi}^{\vee}\right) \stackrel{\oplus m_{\xi}}{\longrightarrow} H^{0}(X, \mathcal{F} \otimes H)
$$

is surjective, where the $m_{\xi}$ denote the multiplication maps on global sections.

Proof. Note that when there is no danger of confusion, we will avoid writing explicitly the names of the maps involved. The conclusion of the theorem is equivalent to the injectivity of the dual map:

$$
H^{0}(\mathcal{F} \otimes H)^{\vee} \longrightarrow \prod_{\xi \in U} H^{0}\left(\mathcal{F} \otimes P_{\xi}\right)^{\vee} \otimes H^{0}\left(H \otimes P_{\xi}^{\vee}\right)^{\vee},
$$

or, by Serre duality (and the fact that $H$ is locally free), with the injectivity of the co-multiplication map:

$$
\operatorname{Ext}^{g}\left(\mathcal{F}, H^{\vee}\right) \longrightarrow \prod_{\xi \in U} \operatorname{Hom}\left(H^{0}\left(\mathcal{F} \otimes P_{\xi}\right), H^{g}\left(H^{\vee} \otimes P_{\xi}\right)\right)
$$

We will concentrate on showing (1). The proof requires the language of derived categories. Let us first reindex, for technical reasons, $R^{j} \hat{\mathcal{S}}(\mathcal{F})$ by $R^{-j} \hat{\mathcal{S}}(\mathcal{F}),-g \leq$ $j \leq 0$. Note also that since $H$ satisfies I.T. with index 0 , by Serre duality $H^{\vee}$ satisfies I.T with index $g$, and so $\widehat{H^{\vee}}=R^{g} \hat{\mathcal{S}}\left(H^{\vee}\right)$ is locally free. We will freely identify $\widehat{H^{\vee}}$ with the one-term complex $\mathbf{R} \hat{\mathcal{S}}\left(H^{\vee}\right)[g]$.

Claim 1. There exists a bounded fourth quadrant cohomological spectral sequence

$$
E_{2}^{i j}=\operatorname{Ext}^{i}\left(R^{-j} \hat{\mathcal{S}}(F), \widehat{H^{\vee}}\right) \Rightarrow H^{i+j}=\operatorname{Ext}_{\mathbf{D}(\hat{X})}^{i+j}\left(\mathbf{R} \hat{\mathcal{S}}(F), \widehat{H^{\vee}}\right) .
$$

Consider the functors $F=\operatorname{Hom}\left(\circ, \widehat{H^{\vee}}\right)$ and $G=\hat{\mathcal{S}}$. We first apply the derived functor $\mathbf{R} G$ to $\mathcal{F}$ to obtain an object in $\mathbf{D}(\hat{X})$. We can then consider a standard hypercohomology spectral sequence (cf. We, 5.7.9 and 10.8.3]:

$$
E_{2}^{i j}=\left(R^{i} F\right)\left(H^{j} \mathbf{R} G(\mathcal{F})\right) \Rightarrow \mathbb{R}^{i+j} F(\mathbf{R} G(\mathcal{F})),
$$

where the $\mathbb{R}^{i} F$ are the right hyperderived functors of $F$ (in this case the hyperexts). This is precisely the sequence in Claim 1 . Note that we have reindexed the $R^{j} \hat{\mathcal{S}}(\mathcal{F})$ in order to agree with the usual sign convention used for the Hom cochain complex We, 2.7.4]. The rest of the assertions are clear.

Claim 2. The spectral sequence in Claim 1 induces a natural inclusion

$$
H^{0+0}=\operatorname{Hom}_{\mathbf{D}(\hat{X})}\left(\mathbf{R} \hat{\mathcal{S}}(\mathcal{F}), \widehat{H^{\vee}}\right) \hookrightarrow E_{2}^{00}=\operatorname{Hom}\left(R^{0} \hat{\mathcal{S}}(\mathcal{F}), \widehat{H^{\vee}}\right) .
$$

Note that the $M$-regularity assumption on $\mathcal{F}$ guarantees that $\operatorname{Ext}^{i}\left(R^{-j} \hat{\mathcal{S}}(\mathcal{F}), \widehat{H^{\vee}}\right)$ $=0$ for $i \leq-j$ and $i>0$, so that the only $E_{\infty}^{i, j}$ term such that $i+j=0$ is $E_{\infty}^{00}$. This implies that

$$
H^{0+0}=\operatorname{Hom}_{\mathbf{D}(\hat{X})}\left(\mathbf{R} \hat{\mathcal{S}}(\mathcal{F}), \widehat{H^{\vee}}\right) \cong E_{\infty}^{00} .
$$

But since we have a fourth quadrant spectral sequence, the differentials coming into $E_{p}^{00}$ are always zero, so we easily get a chain of inclusions

$$
E_{\infty}^{00} \subset \cdots \subset E_{3}^{00} \subset E_{2}^{00} .
$$


Claim 3. There is a natural map of $\mathcal{O}_{\hat{X}}$-modules

$$
\phi: \operatorname{Ext}^{g}\left(\mathcal{F}, H^{\vee}\right) \otimes \mathcal{O}_{\hat{X}} \longrightarrow \mathcal{H o m}\left(R^{0} \hat{\mathcal{S}}(\mathcal{F}), \widehat{H^{\vee}}\right)
$$

such that, for $\xi$ in a suitable open set $V \subset \hat{X}$, the induced maps on the fibers

$$
\phi(\xi): \operatorname{Ext}^{g}\left(\mathcal{F}, H^{\vee}\right) \longrightarrow \mathcal{H o m}\left(R^{0} \hat{\mathcal{S}}(\mathcal{F}), \widehat{H^{\vee}}\right)(\xi)
$$

are the co-multiplication maps in (1).

By Mukai's duality result [M1, Theorem 2.2], we have

$$
\begin{gathered}
\operatorname{Ext}^{g}\left(\mathcal{F}, H^{\vee}\right) \cong \operatorname{Hom}_{\mathbf{D}(X)}\left(\mathcal{F}, H^{\vee}[g]\right) \cong \operatorname{Hom}_{\mathbf{D}(\hat{X})}\left(\mathbf{R} \hat{\mathcal{S}}(\mathcal{F}), \mathbf{R} \hat{\mathcal{S}}\left(H^{\vee}\right)[g]\right) \\
\cong \operatorname{Hom}_{\mathbf{D}(\hat{X})}\left(\mathbf{R} \hat{\mathcal{S}}(\mathcal{F}), \widehat{H^{\vee}}\left[g-i\left(H^{\vee}\right)\right]\right) \cong \operatorname{Hom}_{\mathbf{D}(\hat{X})}\left(\mathbf{R} \hat{\mathcal{S}}(\mathcal{F}), \widehat{H^{\vee}}\right) .
\end{gathered}
$$

We thus get a map at the level of global sections

$$
\Phi=H^{0}(\phi): \operatorname{Ext}^{g}\left(\mathcal{F}, H^{\vee}\right) \longrightarrow \operatorname{Hom}\left(R^{0} \hat{\mathcal{S}}(\mathcal{F}), \widehat{H^{\vee}}\right),
$$

induced by the spectral sequence map in Claim 2. This can be extended in turn to a sheaf map as in the statement of the claim (since the first sheaf is the trivial bundle). It is natural, and not hard to check, that this map coincides with the above co-multiplication maps at the general point.

Finally, $\mathcal{H o m}\left(R^{0} \hat{\mathcal{S}}(\mathcal{F}), R^{g} \hat{\mathcal{S}}\left(H^{\vee}\right)\right.$ ) is a torsion-free sheaf (note that $R^{g} \hat{\mathcal{S}}\left(H^{\vee}\right)$ is locally free), so it is a standard fact that the injectivity of the map in (1) is equivalent to the injectivity of the map $\phi$ of Claim 3 at the $H^{0}$ level, in other words that of the map $\Phi$ in (2). This is precisely the statement of Claim 2, and the proof is completed.

Remark 2.6. If in the theorem above we impose the stronger condition that $\mathcal{F}$ satisfy I.T. with index 0 , then the argument above shows the degeneration of the spectral sequence at the $E_{2}$ level, which implies that the map $\Phi$ is in fact an isomorphism.

Remark 2.7. For the purposes of the present paper we only need Theorem 2.5 in the case when $H$ is an ample line bundle on $X$. There are, however, interesting applications of the full statement, for example, to the study of semihomogeneous vector bundles, which we will describe in [PP2].

Corollary 2.8. Let $\mathcal{F}$ and $H$ be as in the previous theorem. Then there is a positive integer $N$ such that for any general $\xi_{1}, \ldots, \xi_{N} \in \hat{X}$, the map

$$
\bigoplus_{i=1}^{N} H^{0}\left(X, \mathcal{F} \otimes P_{\xi}\right) \otimes H^{0}\left(X, H \otimes P_{\xi}^{\vee}\right) \stackrel{\oplus m_{\xi_{i}}}{\longrightarrow} H^{0}(X, \mathcal{F} \otimes H)
$$

is surjective.

Proof. Let $U$ be an open set of $\hat{X}$ on which the rank of the map $m_{\xi}$ is constant. The surjectivity of the map $\mathcal{M}_{U}$ implies that the family of linear subspaces $\left\{\operatorname{Im}\left(m_{\xi}\right)\right\}_{\xi \in U}$ linearly spans the vector space $H^{0}(\mathcal{F} \otimes H)$. But then there is an $N$ such that $N$ general such subspaces span $H^{0}(\mathcal{F} \otimes H)$.

In the same spirit we have a "preservation of vanishing" statement which will be used in the subsequent sections to deduce vanishing results from $M$-regularity results.

Proposition 2.9. Let $\mathcal{F}$ be an $M$-regular coherent sheaf on $X$ and $H$ a locally free sheaf satisfying I.T. with index 0 . Then $\mathcal{F} \otimes H$ satisfies I.T. with index 0 . 
Proof. For an arbitrary $\alpha \in \operatorname{Pic}^{0}(X)$, since $H$ is locally free we have

$$
H^{i}(\mathcal{F} \otimes H \otimes \alpha) \cong \operatorname{Ext}^{i}\left((H \otimes \alpha)^{\vee}, \mathcal{F}\right) .
$$

Applying again Mukai's duality theorem [M1, Theorem 2.2] we get

$$
\begin{aligned}
& \operatorname{Ext}^{i}\left((H \otimes \alpha)^{\vee}, \mathcal{F}\right)=\operatorname{Hom}_{\mathbf{D}(X)}\left((H \otimes \alpha)^{\vee}, \mathcal{F}[i]\right) \\
& \quad \cong \operatorname{Hom}_{\mathbf{D}(\hat{X})}\left(\mathbf{R} \hat{\mathcal{S}}\left((H \otimes \alpha)^{\vee}\right), \mathbf{R} \hat{\mathcal{S}}(\mathcal{F})[i]\right) \\
& \quad \cong \operatorname{Hom}_{\mathbf{D}(\hat{X})}\left(\left((H \otimes \alpha)^{\vee}\right), \mathbf{R} \hat{\mathcal{S}}(\mathcal{F})[i+g]\right) \cong \operatorname{Ext}_{\mathbf{D}(\hat{X})}^{i+g}\left(\left((H \otimes \alpha)^{\vee}\right), \mathbf{R} \hat{\mathcal{S}}(\mathcal{F})\right) .
\end{aligned}
$$

As in the proof of Theorem 2.5, there is a bounded cohomological spectral sequence

$$
E_{2}^{i j}=\operatorname{Ext}^{i}\left(\left((H \otimes \alpha)^{\vee}\right) \hat{,}, R^{j} \hat{\mathcal{S}}(F)\right) \Rightarrow H^{i+j}=\operatorname{Ext}_{\mathbf{D}(\hat{X})}^{i+j}\left(\left((H \otimes \alpha)^{\vee}\right) \hat{,}, \mathbf{R} \hat{\mathcal{S}}(F)\right) .
$$

Note now that the $M$-regularity assumption on $X$ implies that

$$
\operatorname{Ext}^{i}\left(\left((H \otimes \alpha)^{\vee}\right) \hat{,} R^{j} \hat{\mathcal{S}}(F)\right)=0 \quad \text { for } i+j>g,
$$

so in particular $E_{\infty}^{i, j}=0$ in the same range. This immediately implies that

$$
\operatorname{Ext}_{\mathbf{D}(\hat{X})}^{i+g}\left(\left((H \otimes \alpha)^{\vee}\right) \hat{,} \hat{\mathcal{S}}(\mathcal{F})\right)=0 \quad \text { for } i>0
$$

as claimed.

The fact that Theorem 2.5 produces geometric statements is best explained by introducing the intermediate notion of continuous global generation, which can be in fact defined on an arbitrary irregular variety. As will be clear from the discussion below, a good understanding of this concept provides in turn global generation statements via Proposition 2.12

Definition 2.10. Let $Y$ be an irregular variety. We define a sheaf $\mathcal{F}$ on $Y$ to be continuously globally generated if for any nonempty open subset $U \subset \operatorname{Pic}^{0}(Y)$ the sum of evaluation maps

$$
\bigoplus_{\alpha \in U} H^{0}(\mathcal{F} \otimes \alpha) \otimes \alpha^{\vee} \longrightarrow \mathcal{F}
$$

is surjective.

Remark 2.11. As in Corollary 2.8, this is equivalent to the existence of a number $N$ such that the finite sum of multiplication maps taken over $N$ general line bundles in $\operatorname{Pic}^{0}(Y)$ is surjective. The intuitive significance of this notion comes from the fact that it models precisely the behavior of line bundles on abelian varieties: the "continous system" of all divisors algebraically equivalent to a given one on an abelian variety is "base point free", i.e., the intersection of all divisors in the class is empty. Thus continuous global generation is a generalization to arbitrary coherent sheaves on irregular varieties. Moreover, for line bundles it means precisely the same as in the case of abelian varieties: the intersection of the divisors in $|L \otimes \alpha|$ is empty as $\alpha$ varies over an open subset of $\operatorname{Pic}^{0}(Y)$.

Proposition 2.12. Let $Y$ be a subvariety of an irregular variety $X, \mathcal{F}$ a coherent sheaf and $L$ a line bundle supported on $Y$, both continuously globally generated as sheaves on $X$. Then $\mathcal{F} \otimes L$ is globally generated. 
Proof. We denote the inclusion of $Y$ in $X$ by $i$, but for simplicity we use the same notation $\mathcal{F}$ or $L$ even when we consider them as torsion sheaves on $X$. We then have $H^{0}\left(X, \mathcal{F} \otimes P_{\xi}\right) \cong H^{0}\left(Y, \mathcal{F} \otimes i^{*} P_{\xi}\right)$ and the analogous statement for $L$. Consider the following commutative diagram, obtained by alternating the order of the obvious evaluation and multiplication maps:

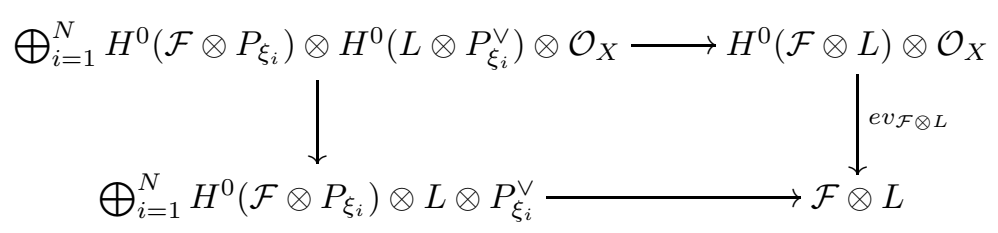

where $N$ is chosen so that for general $\xi_{1}, \ldots, \xi_{N} \in \operatorname{Pic}^{0}(X)$ the bottom horizontal map is onto. It follows that the support of $\operatorname{Coker}\left(e v_{\mathcal{F} \otimes L}\right)$ is contained in the union of the base loci $B\left(L \otimes i^{*} P_{\xi_{i}}^{\vee}\right)$ on $Y$, for $\xi_{1}, \ldots, \xi_{N} \in \operatorname{Pic}^{0}(X)$ general, or equivalently the loci where $L \otimes P_{\xi}^{\vee}$ are not generated by global sections on $X$ individually, since $L$ has rank at most 1 everywhere. But the continuous global generation condition implies precisely that the intersection of these loci is empty (cf. also the remark above), which easily gives that the intersection of such unions is empty.

Finally the key point, using the new language, is that $M$-regular sheaves are always continuously globally generated. More precisely, we have the following:

Proposition 2.13 ( $M$-regularity implies continuous global generation). If $\mathcal{F}$ is $M$-regular, then there is a positive integer $N$ such that for general $\xi_{1}, \ldots, \xi_{N} \in \hat{X}$, the sum of twisted evaluation maps

$$
\bigoplus_{i=1}^{N} H^{0}\left(\mathcal{F} \otimes P_{\xi_{i}}\right) \otimes P_{\xi_{i}}^{\vee} \longrightarrow \mathcal{F}
$$

is surjective.

Proof. We apply Theorem 2.5 with $H$ a line bundle, sufficiently ample so that $\mathcal{F} \otimes H$ is globally generated. As in the proof of Proposition 2.12, consider the commutative diagram:

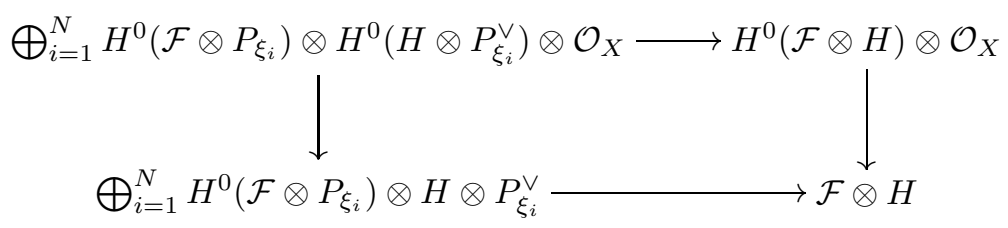

By Corollary 2.8 and the choice of $H$, the top-right composition must be surjective, so the same must hold for the bottom map, which is what we wanted to show.

\section{First eXAmples AND APPlications of $M$-Regularity}

Example 3.1. A line bundle $L$ on a smooth curve $C$ of genus $g \geq 1$ is $M$-regular (via an Abel-Jacobi embedding $C \subset J(C)$ ) if and only if $d=\operatorname{deg} L \geq g$. Indeed, the $M$-regularity of $L$ is equivalent by Riemann-Roch to the fact that $W_{d}^{d-g+1}$ has codimension at least 2 in $\operatorname{Pic}^{d}(C)$. But this is easily seen to be equivalent to $d \geq g$. 
Example 3.2. More generally, let $W_{d} \subset J(C)$ be an Abel-Jacobi embedding given by the choice of a line bundle of degree $d$ on $C$, and consider the natural map $\pi_{d}: C^{d} \rightarrow W_{d}$. Let also $N_{1}, \ldots N_{d}$ be line bundles of degree $g$ on $C$, and on $W_{d}$ consider the sheaf

$$
\mathcal{F}:=\pi_{d *}\left(N_{1} \otimes \cdots \otimes N_{d}\right) .
$$

Then one can see similarly that $\mathcal{F}$ is $M$-regular as a sheaf on $J(C)$. From Theorem 2.4 and Example 3.4 below, one then gets immediately the following

Corollary 3.3. The sheaf $\mathcal{F} \otimes \mathcal{O}_{W_{d}}(\Theta)$ is globally generated on $W_{d}$.

This example is of interest in the sense that it is a priori not clear (at least to us) how to prove this global generation by standard methods. Note that $\mathcal{F} \otimes \mathcal{O}_{W_{d}}(\Theta)$ is the push-forward of the line bundle $\left(N_{1} \otimes \cdots \otimes N_{d}\right) \otimes \pi_{d}^{*} \mathcal{O}(\Theta)$ on $C_{d}$, which is of the form

$$
\left.\left[A \otimes N_{1}\right) \otimes \cdots \otimes\left(A \otimes N_{d}\right)\right] \otimes \mathcal{O}(-\Delta),
$$

where $A$ is a line bundle of degree $g+d-1$ on $C$ and $\Delta$ is the union of the diagonal divisors (cf. $\S 5$ ).

Example 3.4. If $\Theta$ is a theta divisor on the Jacobian of $C$, then $\mathcal{O}_{W_{d}}(\Theta)$ is $M$ regular. This fact will be important in $\S 5$. The proof is not immediate, but see Proposition 4.4.

Linear series - an introduction to [PP1]. In a different direction, our $M$ regularity and global generation results provide a unified approach to very ampleness (in a first stage) statements on abelian varieties and their subvarieties. We will need the following standard lemma.

Lemma 3.5. Let $L$ be a line bundle on a variety $Y$. Then $L$ is very ample if and only if $L \otimes \mathcal{I}_{y}$ is globally generated for all $y \in Y$.

As an immediate consequence of Theorem 2.4 and Lemma 3.5, we get:

Corollary 3.6. Let $Y$ be a subvariety of an abelian variety $X$. Let $L$ and $M$ be line bundles on $Y$ such that $L$ is $M$-regular and $M \otimes \mathcal{I}_{y}$ is $M$-regular for all $y \in Y$, both as sheaves on $X$. Then $L \otimes M$ is very ample.

Lemma 3.7. If $L$ is an ample line bundle with no base divisor on an abelian variety $X$, then $L \otimes \mathcal{I}_{x}$ is $M$-regular for any $x \in X$.

Proof. The cohomological support locus $V^{1}\left(L \otimes \mathcal{I}_{x}\right)$ is the locus of $\xi \in \hat{X}$ corresponding to $P_{\xi} \in \operatorname{Pic}^{0}(X)$ such that $h^{1}\left(L \otimes P_{\xi} \otimes \mathcal{I}_{x}\right) \neq 0$. Since $L \otimes P_{\xi} \otimes \mathcal{I}_{x} \cong$ $t_{\nu}^{*} L \otimes \mathcal{I}_{x} \cong t_{\nu}^{*}\left(L \otimes \mathcal{I}_{x-\nu}\right)$ for some $\nu \in X$, and since $h^{1}(L)=0$, this is precisely isomorphic to the locus where $L$ fails to be globally generated. But now $L$ has no base divisor, so this implies that $V^{1}\left(L \otimes \mathcal{I}_{x}\right)$ has codimension at least 2 . Also the $V^{i}\left(L \otimes \mathcal{I}_{x}\right)$ are obviously empty for $i \geq 2$, so $L \otimes \mathcal{I}_{x}$ is $M$-regular.

Example 3.8. To give a flavor of the range of applications that can be derived as consequences of Corollary 3.6 we explain how some of the most basic facts on curves and abelian varieties are incorporated in this theory. For the abelian varieties statements below cf. [LB, Ch.4, §5].

Corollary 3.9. Let $X$ be an abelian variety and $C$ a smooth projective curve. Then: 
(i) (Lefschetz Theorem) If $L$ is an ample line bundle on $X$, then $L^{\otimes 3}$ is very ample.

(ii) (Ohbuchi's Theorem) If $L$ is an ample line bundle on $X$ with no base divisor, then $L^{\otimes 2}$ is very ample.

(iii) If $L$ is a line bundle of degree at least $2 g+1$ on $C$, then $L$ is very ample.

Proof. By Theorem 3.6 (i) and (ii) follow if we prove that $L^{\otimes 2} \otimes \mathcal{I}_{x}$, respectively $L \otimes \mathcal{I}_{x}$, are $M$-regular. But this is a consequence of Lemma 3.7 since in the first case $L^{\otimes 2}$ is globally generated and in the second case $L$ has no base divisor by assumption. For part (iii) note that a line bundle of degree $2 g+1$ on $C$ can be written as $A \otimes B$, where $A$ has degree $g$ and $B$ has degree $\geq g+1$. By Example 3.1. $A$ and $B(-x)$ are $M$-regular for all $x \in X$, and so again by Theorem 2.4 this implies that $A \otimes B(-x)$ is globally generated.

Remark 3.10. The above corollary is just intended to be an amusing illustration of the fact that well-known and seemingly unrelated results are in fact realizations of the same principle. On the other hand, and more importantly, the proof is a toy version of a general approach, based on the notion of $M$-regularity, which provides a unified treatment of essentially all the known geometric and syzygetic statements on multiples of ample line bundles on abelian varieties, and also produces new basic statements on higher order properties of such embeddings, projective normality and defining equations, as explained in the introduction. This is the main topic of the sequel [PP1] to this paper.

\section{The equations Defining the $W_{d}$ 's in Jacobians AND A CONJECTURE OF OXBURY AND PAULY}

Let $C$ be a smooth curve of genus $g \geq 3$, and denote by $J(C)$ the Jacobian of $C$. Let $\Theta$ be a theta divisor on $J(C)$, and let $C_{d}$ be the $d$-th symmetric product of $C$, for $1 \leq d \leq g-1$. Consider

$$
u_{d}: C_{d} \longrightarrow J(C)
$$

to be an Abel-Jacobi mapping of the symmetric product (depending on the choice of a line bundle of degree $d$ on $C$ ), and denote by $W_{d}$ the image of $u_{d}$ in $J(C)$. In this section we show how the methods developed in this paper allow one to understand the "regularity" of the ideal sheaf $\mathcal{I}_{W_{d}}$ (cf. $\S 6$ for the precise meaning of this). This gives a positive answer to a conjecture of Oxbury and Pauly $\mathrm{OP}$ (cf. Corollary 4.3 below) and bounds in turn the degrees of the Theta-equations defining $W_{d}$ in $J(C)$.

Theorem 4.1. For any $1 \leq d \leq g-1$, the twisted ideal sheaf $\mathcal{I}_{W_{d}}(2 \Theta)$ satisfies I.T. with index 0.

A first application of this result is to defining equations. One would like to know what multiples of $\Theta$ cut out the ideal of $W_{d}$ in $J(C)$, in other words for what $m$ the sheaf $\mathcal{I}_{W_{d}} \otimes \mathcal{O}_{J}(m \Theta)$ is globally generated (or better, continuously globally generated). The answer to this question turns out to be independent of $d$, namely:

Corollary 4.2. For any $1 \leq d \leq g-1$, the ideal $\mathcal{I}_{W_{d}}$ is cut out by divisors algebraically equivalent to $2 \Theta$. Moreover, $\mathcal{I}_{W_{d}}$ is also cut out by divisors linearly equivalent to $3 \Theta$. 
Proof. The first statement means precisely that $\mathcal{I}_{W_{d}}(2 \Theta)$ is continuously globally generated. But this follows immediately from Proposition [2.13] since by Theorem $4.1 \mathcal{I}_{W_{d}}(2 \Theta)$ satisfies I.T. with index 0 , hence it is $M$-regular. On the other hand, by Theorem 2.4 this implies that $\mathcal{I}_{W_{d}}(3 \Theta)$ is globally generated, which gives the second statement.

As mentioned above, Theorem 4.1 also gives an affirmative answer to Conjecture 10.3 in $[\mathrm{OP}]$, stated there in the slightly more restrictive case when $\Theta=\Theta_{\kappa}$, where $\kappa$ is a theta-characteristic on $C$.

Corollary 4.3 (Oxbury-Pauly conjecture). In the notation above the following are true:

(1) $h^{0}\left(C^{d}, u_{d}^{*} \mathcal{O}(2 \Theta)\right)=\Sigma_{i=0}^{d}\left(\begin{array}{c}g \\ i\end{array}\right)$.

(2) $u_{d}^{*}: H^{0}(J(C), \mathcal{O}(2 \Theta)) \rightarrow H^{0}\left(C^{d}, u_{d}^{*} \mathcal{O}(2 \Theta)\right)$ is surjective.

Proof. Both statements follow immediately from the vanishing of $h^{i}\left(\mathcal{I}_{W_{d}} \otimes \mathcal{O}(2 \Theta)\right)$ for all $i>0$, combined with the computation of $\chi\left(u_{d}^{*} \mathcal{O}(2 \Theta)\right)$ given in $\mathrm{OP}$, Proposition 10.1(3)]. But this vanishing (together with that for any other translate) means precisely that $\mathcal{I}_{W_{d}}(2 \Theta)$ satisfies I.T. with index 0 , as stated in Theorem 4.1

A key step in the proof of Theorem 4.1 is the following (cf. also Example 3.4):

Proposition 4.4. (i) The sheaf $\mathcal{O}_{W_{d}}(\Theta)$ is $M$-regular on $J(C)$.

(ii) We have $h^{0}\left(W_{d}, \mathcal{O}_{W_{d}}(\Theta) \otimes P_{\xi}\right)=1$ for $\xi \in \operatorname{Pic}^{0}(J(C))$ generic.

Proof. We will prove the two statements at the same time, by computing explicitly the corresponding cohomology groups. The Abel-Jacobi map has projective spaces as fibers, so the vanishings we are interested in follow as soon as we prove them after pulling back via $u_{d}$. We are then interested in computing the cohomology groups

$$
H^{i}\left(C_{d}, u_{d}^{*} \mathcal{O}(\Theta) \otimes u_{d}^{*} P_{\xi}\right), \quad \forall \xi \in \operatorname{Pic}^{0}(J(C)) .
$$

For this we appeal to the technical results proved in [Iz, Appendix 3.1], which we use freely below. If we denote by $\pi_{d}: C^{d} \rightarrow J(C)$ the corresponding desymmetrized Abel-Jacobi map, we have:

$$
\pi_{d}^{*} \mathcal{O}(\Theta) \cong(A \otimes \cdots \otimes A) \otimes \mathcal{O}(-\Delta),
$$

where $\Delta$ is the union of all the diagonal divisors in $C^{d}$ and $A$ is a line bundle of degree $g+d-1$. We then have the identification:

$$
H^{i}\left(u_{d}^{*} \mathcal{O}(\Theta) \otimes u_{d}^{*} P_{\xi}\right) \cong S^{i} H^{1}(C, A \otimes \xi) \otimes \wedge^{d-i} H^{0}(C, A \otimes \xi),
$$

obtained as the skew-symmetric part of the cohomology group $H^{i}\left((A \otimes \xi)^{\otimes d}\right)$ under the action of $S_{d}$. Now we only have to compute the loci on which these cohomology groups do not vanish, for each $0 \leq i \leq d$. Since $\xi$ is a general line bundle on $C$ of degree $0, A \otimes \xi$ is a general line bundle of degree $g+d-1$. Thus statement (ii) follows immediately, since $H^{1}(C, A \otimes \xi)=0$ and so

$$
H^{0}(C, A \otimes \xi)=d .
$$

On the other hand, for $i>0$, if $H^{i}\left(u_{d}^{*} \mathcal{O}(\Theta) \otimes u_{d}^{*} P_{\xi}\right)$ is nonzero, then $h^{1}(A \otimes \xi)=$ $h^{0}\left(\omega_{C} \otimes A^{-1} \otimes \xi^{-1}\right) \neq 0$. This locus is isomorphic to $W_{g-d-1}$, and so it is of codimension $d+1$, which gives (i). 
Proof of Theorem 4.1. From the standard cohomology sequence associated to the exact sequence

$$
0 \longrightarrow \mathcal{I}_{W_{d}}(2 \Theta) \longrightarrow \mathcal{O}_{J(C)}(2 \Theta) \longrightarrow \mathcal{O}_{W_{d}}(2 \Theta) \longrightarrow 0,
$$

we see that the theorem follows as soon as we prove the following two statements, where this time for simplicity we denote by $\Theta$ any of its translates:

- $H^{i}\left(\mathcal{O}_{W_{d}}(2 \Theta)\right)=0, \forall i>0$.

- The restriction map $H^{0}\left(\mathcal{O}_{J(C)}(2 \Theta)\right) \rightarrow H^{0}\left(\mathcal{O}_{W_{d}}(2 \Theta)\right)$ is surjective.

The first statement means precisely that $\mathcal{O}_{W_{d}}(2 \Theta)$ satisfies I.T. with index 0, which follows immediately from Proposition 4.4(i) and Proposition 2.9]

For the second statement we will appeal directly to Theorem [2.5 Note that for any open subset $U \in \widehat{J(C)}$ we have a commutative diagram as follows, where the vertical maps are the natural restrictions.

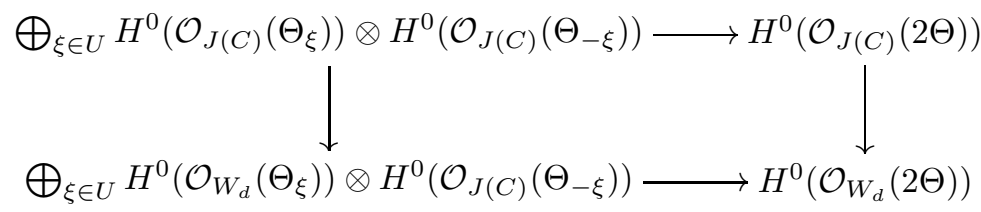

Now Theorem 2.5 says that the bottom horizontal map is surjective. On the other hand, by Proposition 4.4(ii) we can choose the open set $U$ such that the left vertical map is an isomorphism. (Note that we cannot include all the translates $\Theta_{\xi}$ such that $h^{0}\left(\mathcal{O}_{W_{d}}\left(\Theta_{\xi}\right)\right)=1$, but certainly those that satisfy this property plus the fact that $W_{d}$ is not contained in $\Theta_{\xi}$.) This in turn implies that the right vertical map is surjective, which is precisely our statement.

\section{Applications Via VANishing TheOREMS}

In combination with various vanishing theorems, like those of KawamataViehweg, Kollár or Nadel, the $M$-regularity criterion produces effective geometric results on linear series and special coherent sheaves on subvarieties of abelian varieties and more general irregular varieties, in the spirit of [Ko2 . We assume here that we are working over a field of characteristic zero. In all the applications below, $M$-regularity will be satisfied in the strong form of I.T. with index 0 .

Effective global generation and very ampleness. The following result treats the case of (finite covers of) subvarieties of abelian varieties (but see also Remark 5.3 below).

Theorem 5.1. (i) Let $Y$ be a smooth irregular variety whose Albanese map is finite onto its image and let $L$ be a big and nef line bundle on $Y$. Then $\omega_{Y}^{\otimes 2} \otimes L^{\otimes 2}$ is globally generated. In particular, if $Y$ is in addition a minimal variety of general type, then $\omega_{Y}^{\otimes 4}$ is globally generated.

(ii) Under the same hypotheses, $\omega_{Y}^{\otimes 3} \otimes L^{\otimes 3}$ is very ample. In particular, if $Y$ is also a minimal variety of general type, then $\omega_{Y}^{\otimes 6}$ is very ample.

Proof. (i) By Proposition 2.12, the result follows if we prove that $\omega_{Y} \otimes L$ is continuously globally generated. As with global generation, it is easy to see that the continuous global generation of $\omega_{Y} \otimes L$ is implied by that of $\operatorname{alb}_{Y *}\left(\omega_{Y} \otimes L\right)$, since 
$a b_{Y}$ is finite. The Kawamata-Viehweg vanishing theorem on $Y$, and again the fact that alb $_{Y}$ is finite, imply that

$h^{i}\left(\operatorname{alb}_{Y *}\left(\omega_{Y} \otimes L\right) \otimes \alpha\right)=h^{i}\left(\omega_{Y} \otimes L \otimes \alpha\right)=0, \forall i>0, \forall \alpha \in \operatorname{Pic}^{0}(Y)=\operatorname{Pic}^{0}(\operatorname{Alb}(Y))$.

This means that $\operatorname{alb}_{Y *}\left(\omega_{Y} \otimes L\right)$ satisfies I.T. with index 0 , hence it is continuously globally generated by Proposition 2.13

(ii) By Lemma 3.5 Proposition 2.12 and the fact that $\omega_{Y} \otimes L$ is continuously globally generated, it would be enough to show that $\omega_{Y}^{\otimes 2} \otimes L^{\otimes 2} \otimes \mathcal{I}_{y}$ is continuously globally generated for all $y \in Y$. As above, this is implied by the same statement for the push-forward to $\operatorname{Alb}(Y)$, which in turn follows as long as $\operatorname{alb}_{Y *}\left(\omega_{Y}^{\otimes 2} \otimes L^{\otimes 2} \otimes \mathcal{I}_{y}\right)$ satisfies I.T. with index 0 . But this is immediate, since as $\omega_{Y} \otimes L$ is continuously globally generated, it is nef and so $\omega_{Y} \otimes L^{\otimes 2}$ is big and nef. Consequently,

$$
H^{i}\left(\omega_{Y}^{\otimes 2} \otimes L^{\otimes 2} \otimes \mathcal{I}_{y} \otimes \alpha\right)=0
$$

(for all $\alpha \in \operatorname{Pic}^{0}(Y)$ ), trivially for $i \geq 2$, and because of the global generation of (deformations of) $\omega_{Y}^{\otimes 2} \otimes L^{\otimes 2}$ for $i=1$.

Remark 5.2. The result above can be stated more generally for Gorenstein varieties with rational singularities, on which the vanishing theorem still applies.

Remark 5.3. Although we have preferred to state the result above in the most compact form, it is important to mention that the same statments hold (and with essentially the same argument) in the more naturally occurring situation of varieties of maximal Albanese dimension, at least outside the exceptional loci, i.e., outside the higher dimensional fibers of the generically finite Albanese map.

The point in the above is that given a big and nef line bundle $L$ on an irregular variety $Y$ with finite Albanese map, $\omega_{Y} \otimes L$ is continuously globally generated. This implies in particular both that it is nef and that it has global sections. Consequently this gives a positive answer to Kawamata's nonvanishing conjecture [Ka2 on varieties of this kind (note that the nefness hypothesis in the conjecture is superfluous in this case).

Corollary 5.4. Let $Y$ be a smooth irregular variety with finite Albanese map and let $L$ be a big and nef line bundle on $Y$. Then $H^{0}\left(\omega_{Y} \otimes L\right) \neq 0$.

The proof of Theorem 5.1 produces also a similar result depending this time on a fixed arbitrary polarization on the target abelian variety.

Proposition 5.5. Let $f: Y \rightarrow X$ be a finite morphism from a projective variety to a polarized abelian variety $(X, \Theta)$, and let $\mathcal{F}$ be a coherent sheaf on $Y$. If $f_{*} \mathcal{F}$ is $M$-regular, then $\mathcal{F} \otimes f^{*} \mathcal{O}_{X}(\Theta)$ is globally generated. In particular, if $Y$ is smooth and $L$ is big and nef on $Y$, then $\omega_{Y} \otimes L \otimes f^{*} \mathcal{O}_{X}(\Theta)$ is globally generated and $\omega_{Y}^{\otimes 2} \otimes L^{\otimes 2} \otimes f^{*} \mathcal{O}_{X}(\Theta)$ is very ample, where $\Theta$ is an arbitrary polarization on $X$.

On a slightly different note, Fujita's problem on the global generation of adjoint bundles has a relative version, which we learned of from [Ka1]. Since on abelian varieties the square of an ample line bundle is always globally generated, it is natural to ask whether there is an analogous uniform statement in the relative setting. This again follows quickly from Theorem 2.4

Proposition 5.6. Let $f: Y \rightarrow X$ be a proper, surjective morphism from a smooth variety $Y$ to an abelian variety $X$, and denote by $\omega_{Y}$ the canonical line bundle on $Y$. Then $f_{*} \omega_{Y} \otimes \mathcal{O}_{X}(2 \Theta)$ is globally generated, for any ample divisor $\Theta$ on $X$. 
Proof. This follows from Theorem 2.4 via a special case of Kollár's theorem on direct images of dualizing sheaves [Ko1], which states that

$$
h^{i}\left(f_{*} \omega_{Y} \otimes L\right)=0, \forall i>0,
$$

for any ample line bundle $L$ on $X$.

An optimal Mumford type lemma for multiplier ideals. Vanishing theorems for coherent sheaves often lead to global generation statements via the CastelnuovoMumford Lemma (cf. §6). In particular, a general such statement for multiplier ideal sheaves is getting to be known as Mumford's Lemma. This has found some very important applications, for example, in Siu's proof of the deformation invariance of plurigenera or to Fujita type statements on the volume of big divisors (cf. [La1, §4.3] for a general discussion). We show here that on abelian varieties one can do substantially better: the analogy to keep in mind is the difference between the general Fujita type statements and the best possible global generation bounds on abelian varieties (like the global generation of $\mathcal{O}_{X}(2 \Theta)$ ). For the general theory of multiplier ideals we refer the reader to [La1.

Let $D$ be an effective $\mathbb{Q}$-divisor on $X$ and denote by $\mathcal{J}(D)$ the associated multiplier ideal sheaf.

Proposition 5.7. If $L$ is a divisor on $X$ such that $L-D$ is ample, then the sheaf $\mathcal{O}_{X}(\Theta+L) \otimes \mathcal{J}(D)$ is globally generated.

Proof. By Theorem [2.4, it is sufficient to have the vanishing:

$$
h^{i}\left(\mathcal{O}_{X}(L) \otimes \mathcal{J}(D) \otimes \alpha\right)=0, \forall i>0, \forall \alpha \in \operatorname{Pic}^{0}(X) .
$$

Now by assumption $\mathcal{O}_{X}(L-D) \otimes \alpha$ is ample (and of course $\omega_{X}=\mathcal{O}_{X}$ ), so this follows from the Nadel vanishing theorem (cf. [La1, Theorem 4.6].

Although not in direct relationship with our criterion, in order to complete the picture we note also that the abelian varieties variant of the nonvanishing theorem for multiplier ideals [La1, §4.3] holds in a very strong form.

Proposition 5.8. Under the assumptions of Proposition 5.7

$$
H^{0}\left(\mathcal{O}_{X}(L) \otimes \mathcal{J}(D)\right) \neq 0 .
$$

Proof. Since $\mathcal{O}_{X}(L-D) \otimes \alpha$ is ample for all $\alpha \in \operatorname{Pic}^{0}(X)$, the Nadel vanishing theorem implies that $\mathcal{O}_{X}(L) \otimes \mathcal{J}(D)$ satisfies I.T. with index 0. By Proposition 2.13 it is then continuously globally generated, so it has sections.

One can formulate analogous statements for multiplier ideals of $\mathbb{Q}$-divisors on smooth subvarieties of abelian varieties. One such version, whose proof is essentially that of Proposition [5.7 together with Proposition 2.12 can be stated as follows:

Proposition 5.9. Let $Y$ be a smooth subvariety of $X$ and $D$ an effective $\mathbb{Q}$-divisor on $Y$. Assume that $L$ is a divisor such that $L-D$ is big and nef, and $M$ is a continuously globally generated divisor on $Y$. Then the sheaf

$$
\mathcal{O}_{Y}\left(K_{Y}+L+M\right) \otimes \mathcal{J}(D)
$$

is globally generated. 


\section{Theta Regularity and an "Abelian" Castelnuovo-Mumford Lemma}

The notion of $M$-regularity might not be an a priori obvious analogue of the usual notion of regularity on projective spaces. There is however a particular instance of the general theory, depending on a fixed polarization $\Theta$, which is more intuitive and is already responsible for some of the applications above. We will call this Theta regularity in what follows. The point of this section is to see that the theory of Theta regularity is strikingly similar to the theory of Castelnuovo-Mumford regularity for coherent sheaves on (subvarieties of) projective spaces.

Definition 6.1. A coherent sheaf $\mathcal{F}$ on a polarized abelian variety $(X, \Theta)$ is called $m$ - $\Theta$-regular if $\mathcal{F}((m-1) \Theta)$ is $M$-regular. If $\mathcal{F}$ is 0 - $\Theta$-regular, we will simply call it $\Theta$-regular.

Example 6.2. Many of the results of the previous two sections can be phrased in the language of Theta regularity. For example, $\mathcal{O}_{W_{d}}$ is $2-\Theta$-regular, while Theorem 4.1 implies that $\mathcal{I}_{W_{d}}$ is 3 - $\Theta$-regular.

We collect in the following theorem a number of results which show the similarity mentioned above. This can be considered a Theta-regularity version of the Castelnuovo-Mumford Lemma. For the usual Castelnuovo-Mumford Lemma cf., e.g., Mu3, Lecture 14].

Theorem 6.3. Let $\mathcal{F}$ be a $\Theta$-regular coherent sheaf on $X$. Then:

(1) $\mathcal{F}$ is globally generated.

(2) $\mathcal{F}$ is $m$ - $\Theta$-regular for any $m \geq 1$.

(3) The multiplication map

$$
H^{0}(\mathcal{F}(\Theta)) \otimes H^{0}(\mathcal{O}(k \Theta)) \longrightarrow H^{0}(\mathcal{F}((k+1) \Theta))
$$

is surjective for any $k \geq 2$.

Proof. Part (1) is a particular case of Theorem 2.4 while (2) follows immediately from Proposition 2.9, as $\mathcal{O}(\Theta)$ satisfies I.T. with index 0. For (3) note that, for any open subset $U \subset \hat{X}$, we have a commutative diagram

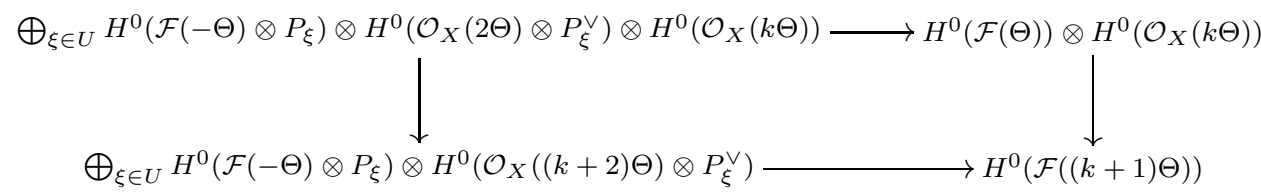

Now the left vertical map is surjective if $U$ is chosen such that Ohbuchi's projective normality theorem (cf. [Oh]) applies for any $\xi \in U$. In addition, since $\mathcal{F}(-\Theta)$ is assumed to be $M$-regular, the bottom horizontal map is also surjective by Theorem 2.5. This implies that the right vertical map is surjective.

Remark 6.4. The numerical analogy with Castelnuovo-Mumford regularity is not perfect in part (3) of Theorem 6.3. It is easy to see though that the statement in (3) is optimal, as it follows, for example, by considering $\mathcal{F}$ equal to $\mathcal{O}(2 \Theta)$ (when we cannot make $k=1$ ). This particular choice also shows that this "abelian" Castelnuovo-Mumford statement contains the well-known Mumford type projective normality results for multiples of ample line bundles (cf. Mu2]). Note also that in this form part (3) generalizes to Theta-regular coherent sheaves a weaker statement about locally free sheaves satisfying I.T. with index 0, which follows as a consequence of $[\mathrm{Pa}$, Theorem 3.8]. 
Bounds on $\Theta$-regularity in terms of defining equations. The CastelnuovoMumford regularity of a subvariety in projective space is a measure of the complexity of the computations involving its ideal (cf., e.g., $[\mathrm{BM}]$ ). It is natural to ask for bounds on this invariant in terms of the degrees of defining equations for the subvariety. This has been optimally achieved for smooth subvarieties of projective spaces in BEL. Completely similar arguments (which we will not repeat here) can be used to give a bound on the $\Theta$-regularity of a smooth subvariety of an abelian variety in terms of the degrees of its pluritheta generators. This can be quite naturally seen as a converse of the cohomological global generation criterion Theorem 6.3(1) in the case of ideal sheaves of smooth subvarieties.

Theorem 6.5. Let $Y$ be a codimension e smooth subvariety of the polarized abelian variety $(X, \Theta)$, and assume that the ideal of $Y$ in $X$ is cut out by $d \Theta$-equations (i.e., the sheaf $\mathcal{I}_{Y} \otimes \mathcal{O}_{X}(d \Theta)$ is globally generated). Then $\mathcal{I}_{Y}$ is ed- $\Theta$-regular, more precisely, $\mathcal{I}_{Y} \otimes \mathcal{O}_{X}((e d+1) \Theta)$ satisfies I.T. with index 0 . Moreover, the $\Theta$-regularity of $\mathcal{I}_{Y}$ is precisely ed if and only if $Y$ is a complete intersection of $d \Theta$-hypersurfaces in $X$.

Theorem 6.5 also has a more general version, where the degrees of the defining $\Theta$-equations are allowed to vary, along the lines of [BEL, Corollary 4].

\section{ACKNOWLEDGEMENTS}

Special thanks are due to $\mathrm{R}$. Lazarsfeld, whose suggestions at various stages have improved both authors' way of thinking about abelian varieties. We are also indebted to I. Dolgachev and B. van Geemen for some useful conversations, to C. Pauly for drawing our attention to the conjecture in $[\overline{\mathrm{OP}}$, and to J.A. Chen and C.D. Hacon for showing us their preprint $[\mathrm{CH}]$ and for a remark improving the statement of Theorem 5.1(ii). The second author would like to thank B. van Geemen, K. O'Grady and University of Rome I for hospitality and financial support during a visit when this project was started. Finally, we thank the referee for careful suggestions which have allowed us to fix some mistakes and substantially improve the exposition.

\section{REFERENCES}

[BM] D. Bayer and D. Mumford, What can be computed in algebraic geometry?, Computational algebraic geometry and commutative algebra (Cortona, 1991), Cambridge Univ. Press (1993), 1-48. MR 95d:13032

[BEL] A. Bertram, L. Ein and R. Lazarsfeld, Vanishing theorems, a theorem of Severi, and the equations defining projective varieties, J. Amer. Math. Soc. 4 (1991), no.3, 587-602. MR 92g:14014

$[\mathrm{CH}]$ J.A. Chen and C.D. Hacon, Effective generation of adjoint linear series of irregular varieties, preprint (2001).

[GL] M. Green and R. Lazarsfeld, Deformation theory, generic vanishing theorems, and some conjectures of Enriques, Catanese and Beauville, Invent. Math. 90 (1987), 389-407. MR 89b:32025

[Iz] E. Izadi, Deforming curves representing multiples of the minimal class in Jacobians to non-Jacobians I, preprint mathAG/0103204.

[Ka1] Y. Kawamata, Semipositivity, vanishing and applications, Lectures at the ICTP Summer School on Vanishing Theorems, April-May 2000.

[Ka2] Y. Kawamata, On effective non-vanishing and base-point-freeness, Asian J. Math 4 (2000), 173-181. MR 2002b:14010 
[Ke1] G. Kempf, On the geometry of a theorem of Riemann, Ann. of Math. 98 (1973), 178-185. MR 50:2180

[Ke2] G. Kempf, Projective coordinate rings of abelian varieties, in: Algebraic Analysis, Geometry and Number Theory, J.I. Igusa ed., Johns Hopkins Press (1989), 225-236. MR 98m:14047

[Ke3] G. Kempf, Complex abelian varieties and theta functions, Springer-Verlag, 1991. MR 92h:14028

[Ko1] J. Kollár, Higher direct images of dualizing sheaves I, Ann. of Math. 123 (1986), 11-42. MR 87c:14038

[Ko2] J. Kollár, Shafarevich maps and automorphic forms, Princeton Univ. Press, 1995. MR 96i: 14016

[LB] H. Lange and Ch. Birkenhake, Complex abelian varieties, Springer-Verlag, 1992. MR 94j:14001

[La1] R. Lazarsfeld, Multiplier Ideals for Algebraic Geometers, notes available at www.math.lsa.umich.edu/ rlaz, to be included in Positivity in Algebraic Geometry, book in preparation.

[La2] R. Lazarsfeld, A sharp Castelnuovo bound for smooth surfaces, Duke Math. J. 55 (1987), 423-428. MR 89d:14007

[M1] S. Mukai, Duality between $D(X)$ and $D(\widehat{X})$ with its application to Picard sheaves, Nagoya Math. J. 81 (1981), 153-175. MR 82f:14036

[M2] S. Mukai, Fourier functor and its application to the moduli of bundles on an abelian variety, In: Algebraic Geometry, Sendai 1985, Advanced studies in pure mathematics 10 (1987), 515-550. MR 89k:14026

[Mu1] D. Mumford, Abelian varieties, Second edition, Oxford Univ. Press, 1974. MR 44:219

[Mu2] D. Mumford, On the equations defining abelian varieties, Invent. Math. 1 (1966), 287-354. MR 34:4269

[Mu3] D. Mumford, Lectures on curves on an algebraic surface, Princeton University Press, 1966. MR 35:187

[Oh] A. Ohbuchi, A note on the normal generation of ample line bundles on abelian varieties, Proc. Japan Acad. 64 (1988), 119-120. MR 90a:14062a

[OP] W. Oxbury and C. Pauly, Heisenberg invariant quartics and $S U_{C}(2)$ for a curve of genus four, Math. Proc. Camb. Phil. Soc. 125 (1999), 295-319. MR 99k:14022

$[\mathrm{Pa}]$ G. Pareschi, Syzygies of abelian varieties, J. Amer. Math. Soc. 13 (2000), 651-664. MR 2001f: 14086

[PP1] G. Pareschi and M. Popa, Regularity on abelian varieties II: basic results on linear series and defining equations, preprint math.AG/0110004.

[PP2] G. Pareschi and M. Popa, in preparation.

[We] Ch. Weibel, An introduction to homological algebra, Cambridge Univ. Press, 1994. MR 95f: 18001

Dipartamento di Matematica, Università di Roma, Tor Vergata, V.le della Ricerca Scientifica, I-00133 Roma, Italy

E-mail address: pareschi@mat.uniroma2.it

Department of Mathematics, Harvard University, One Oxford Street, Cambridge, MASSACHUSETtS 02138

E-mail address: mpopa@math.harvard.edu 